Gibson, G., and Norymberski, J. K. (1954). Annals of the Rheumatic Diseases, 13, 59 . Hartog, M., Joplin, G. F., Fotherby, K., Mattingly, D., and Fraser, T. R.
(1968). In Investigation of Hypothalamic-Pituitary-Adrenal Function ed. V. H. T. James and J. Landon, p. 271. London, Cambridge University Press.

James, V. H. T., Mattingly, D., and Daly, J. R. (1971). British Medical fournal, 2,310 .

Liddle, G. W. (1960). Fournal of Clinical Endocrinology and Metabolism, 20,1539 .

McHardy-Young, S., Harris, P. W. R., Lessof, M. H., and Lyne, C. (1967). British Medical fournal, 2,740

Mattingly, D. (1962). Fournal of Clinical Pathology, 15, 374.

Mattingly, D. (1963). Proceedings of the Royal Society of Medicine, 56, 717.

Mattingly, D. (1968). In Recent Advances in Medicine, ed. D. N. Baron, N. Compton, and A. M. Dawson, p. 157. London, Churchill.
Mattingly, D., Dennis, P. M., Pearson, J., and Cope, C. L. (1964). Lancet 2, 1046 .

Mattingly, D., and Tyler, C. M. (1967). British Medical fournal, 4, 394.

Nugent, C. A., Nichols, T., and Tyler, F. H. (1965). Archives of Internal Medicine, 116, 172.

Ross, E. J., Marshall-Jones, P., and Friedman, M. (1966). Quarterly fournal of Medicine, 35, 149

Seidensticker, J. F., Folk, R. L., Wieland, R. G., and Hamwi, G. J. (1967). Fournal of the American Medical Association, 202, 87

Slater, J. D. H., Hartog, M., Fraser, R., and Rantzen, B. (1962). British Medical fournal, 1, 1584

Summers, V. K., Sheehan, H. L., Hipkin, L. J., and Davis, J. C. (1964). Lancet, 2, 1079.

Symington, T. (1969). Functional Pathology of the Human Adrenal Gland, p. 109. Edinburgh, Livingstone.

\title{
In-vivo Cytogenetic Effects of Perphenazine and Chlorpromazine: A Negative Study
}

\author{
MAIMON M. COHEN, ERNEST LIEBER, HOWARD N. SCHWARTZ
}

British Medical fournal, 1972, 3, 21-23

\section{Summary}

No evidence was found that either perphenazine or chlorpromazine induced chromosomal damage in patients given the drugs. These results were reached independently at two separate laboratories; earlier reports of possible adverse cytogenetic effects of these drugs can probably be discounted.

\section{Introduction}

During the past few years the cytogenetic effects of an increasing number of psychotropic agents have been investigated (Cohen et al., 1967; Cohen et al., 1969; Nielsen et al., 1969; Schmid and Staiger, 1969; Staiger, 1969; Stenchever and Frankel, 1969). Simple explanations for the apparent conflicting observations obtained with some drugs are exceedingly difficult to propose, but such factors as observer differences and differences in tissue culture medium, culture time, and technique have been suggested. This report assesses the possible induction of in-vivo chromosomal damage by two psychotropic agents (perphenazine and chlorpromazine) which have previously been implicated as "chromosome breakers" (Cohen et al., 1967; Nielsen et al., 1969). The experiment was designed to test for differences between the procedures used in two different cytogenetic laboratories.

\section{Materials and Methods}

Nineteen drug-treated male subjects aged 23-63 years were studied. They were patients at the Neuropsychiatric Institute, Princeton, New Jersey. All had histories of chronic schizophrenic reactions of various types, and all had received daily doses of standard and investigational drugs for up to two years before the experiment began.

\footnotetext{
Department of Pediatrics, Buffalo Children's Hospital, Buffalo, New York 14222, U.S.A.

MAIMON M. COHEN, PH.D., Associate Professor of Pediatrics and Director of Cytogenetics

Beth Israel Hospital, New York, U.S.A.

ERNEST LIEBER, M.D., Chief, Medical Genetics Unit

Schering Corporation, Bloomfield, New Jersey, U.S.A.

HOWARD N. SCHWARTZ, M.D., Director, Department of Clinical Pharmacology
}

Perphenazine was given to nine patients in increasing doses over a six-week period. Daily doses were increased every two days, ranging from $8 \mathrm{mg} /$ day to a maximum of $48 \mathrm{mg} /$ day. The average total dose per patient was $1,110 \mathrm{mg}$ during the six-week period.

Chlorpromazine was given to 10 patients. The daily dose range was increased from $150 \mathrm{mg}$ to $600 \mathrm{mg} /$ day at about two-day intervals during the six-week study. Average total dose per patient was $18,565 \mathrm{mg}$.

Six normal males were selected as controls from hospital personnel with no history of previous drug ingestion who worked on the same wards as the patients.

During the course of this study none of the volunteers or patients needed radiological procedures or suffered any intercurrent illness or viral infections.

Experimental Design.-Drugs were discontinued in all cases for one month before the study began (washout, period 1). Blood samples were obtained for chromosome analysis at the beginning of the washout phase and again at its completion (baseline, period 2). Similar samples were then obtained after three (period 3) and six (period 4) weeks of daily drug ingestion. At the completion of period 4 all drugs were withheld for up to one month, and a final blood sample was obtained (period 5) before reinstitution of therapy.

Chromosome Preparations. - Blood samples were collected in heparinized syringes which were numerically coded. Three to five drops of whole blood was inoculated into each of four microculture tubes (chromosome medium 1A, GIBCO). Two tubes were sent to each of the participating cytogenetics laboratories (New York City and Buffalo) for culture and analysis. The same batch of medium was used throughout the study. Similar procedures of culture, harvesting, slide preparation, and scoring were used in both laboratories. The cultures were incubated at $37^{\circ} \mathrm{C}$ for 72 hours, the final two hours in the presence of Colcemid (demecolcine) $(0.5 \mu \mathrm{g} / \mathrm{ml})$ or Velban (vinblastine sulphate) $(0.05 \mu \mathrm{g} / \mathrm{ml})$. The cultures were harvested after a slight modification of the method of Moorhead et al. (1960). After centrifugation the supernate was discarded and the cells were resuspended in hypotonic sodium citrate $(1 \%)$ or fetal calf serum in distilled water $(1: 5)$ for $15 \mathrm{~min}$ at $37^{\circ} \mathrm{C}$. The cells were then fixed in several changes (10 min each) of the $3: 1$ methanol: glacial acetic acid fixative. Two drops of cell suspension were then placed on a chilled, prewet (either distilled water or $70 \%$ methanol) microscope slide and passed through a flame for ignition. The slides were stained in $2 \%$ acetic orcein or Giemsa and dehydrated in a graduated series of alcohol, and a coverslip was mounted with Permount. 
Chromosome Scoring.-For each blood sample obtained four slides were prepared (two per culture tube) and scored for various abnormalities, including chromosome breaks, gaps, structural abnormalities (rings, dicentrics, triradials, quadriradials), and mitotic rate. Twenty-five cells per slide (total 100 per sample) were selected under low power $(\times 250)$ and subsequently examined under oil immersion $(\times 1,650)$ for cytogenetic damage. A mitotic index was ascertained by scoring the number of mitoses seen in 250 cells on each slide (total 1,000). Chromosomal damage was classified as previously described (Cohen et al., 1967). Both chromatid and isochromatid breaks were scored as single anomalies. Exchange figures, rings, and dicentric chromosomes were counted as two breaks each. Gaps or visual discontinuities of the chromatin material without displacement of the distal portion were noted but not regarded as breaks.

Statistical Analysis. - Statistical evaluation was carried out via a one-way covariance analysis by using the predrug reading (baseline, period 2) for each variable as the covariate. Analyses were performed for each laboratory separately as well as on the combined results. Advantages of the covariance approach are its ability to adjust for any initial differences in the response variable and to reduce the amount of unexplained variation by use of the covariate.

\section{Results}

Results obtained in this experiment showed no significant differences between the controls and the drug-treated patients during the entire course of the study. Moreover, the investigations performed independently in the two laboratories did not differ statistically. Since there were no significant differences within any given group of patients nor between laboratories the figures in the Table represent the means and standard errors for the particular treatment group at the various periods of examination.

The data on total abnormal cells given in the Table include all abnormalities-for example, gaps, simple breaks, and rearrangements. No significant differences were noted among the various drug groups or between laboratories $(P>0.25)$. The findings were similar for the various individual abnormalities scored-that is, gaps alone $(P>0.25)$ and breaks $(P>0.25)$. A total of 14 structural rearrangements were observed-seven dicentrics, three rings, and four triradials and quadriradials (see Fig.). These abnormalities were seen in both controls and treated patients.

There was no difference in the rate of cell division between the patients and the controls (see Table). The mitotic rates decreased (although not significantly) at the last examination after a

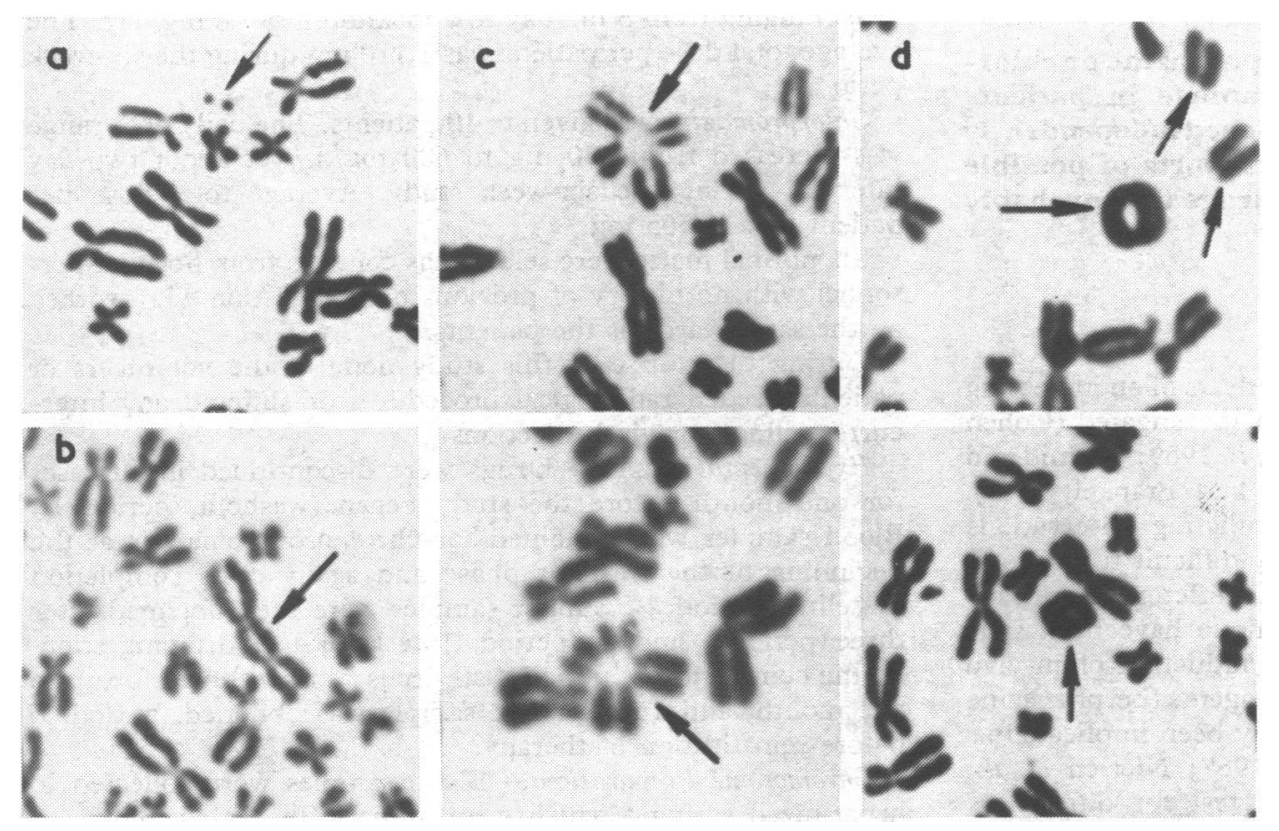

Chromosome damage found in subjects studied. a, Arrow indicates "double" fragment resulting from isochromatid break. b, Dicentric chromosome. c, (upper) asymmetrical (lower). d, Ring chromosomes; upper frame include ${ }^{2}$ additional acentric fragments (arrowed).

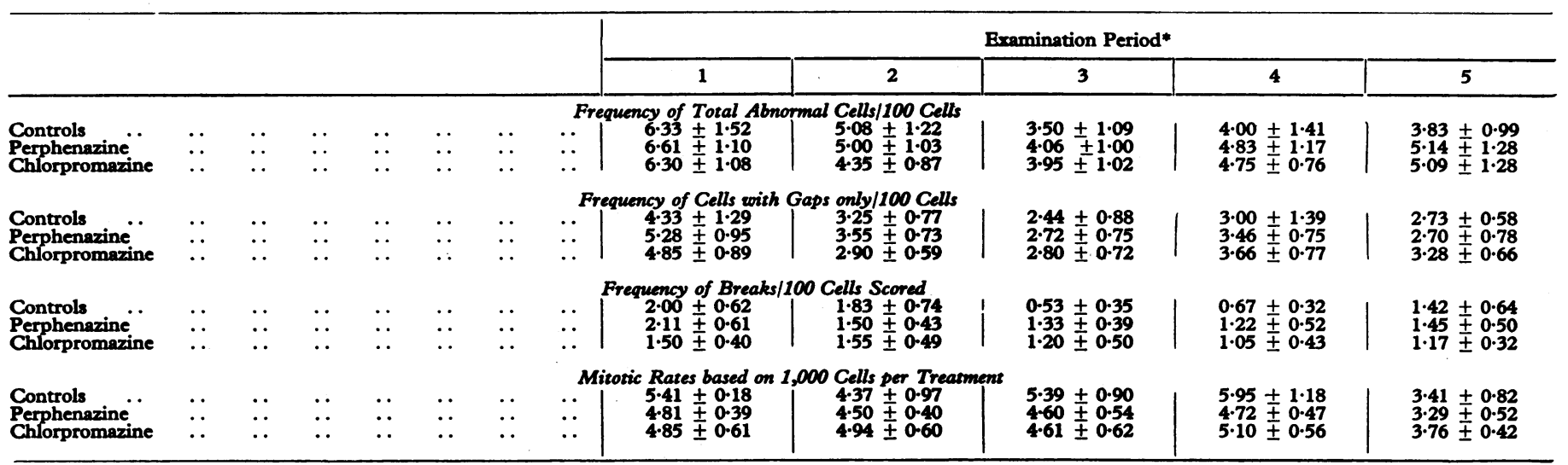

- For details see text. 
one-month period without medication. This reduction, however, was apparent in the controls as well as in the patients receiving the drugs and was obvious in both laboratories.

\section{Discussion}

Reports of the chromosome-breaking potential of various drugs have evoked much concern, with particular attention paid to those psychotropic compounds (including hallucinogens) reportedly having this capability. Nonetheless, relatively few well-controlled in-vivo prospective studies have been performed to investigate these properties. In the present investigation an attempt was made to obtain baseline values of chromosome damage in the subjects before the actual investigation began. An additional feature of this double-blind study was the simultaneous culturing and scoring of replicate samples from each subject in two independent cytogenetic laboratories. The results were completely negative for both drugs tested and there was no statistically significant difference between the laboratories for any value scored. The method of culturing, harvesting, and scoring of the cells was similar although not identical in both laboratories, and therefore results tested the repeatability and reliability of such procedures.

Our negative findings indicate that neither perphenazine nor chlorpromazine has chromosome-breakage potential. This is in contrast to the findings of Nielsen et al. (1969), which indicated that perphenazine could perhaps induce chromosome damage. That study, however, contained several possible biases. Firstly, no systematic attempt was made to obtain baseline values of chromosome damage in the subjects studied. More importantly, those patients receiving perphenazine also received orphenadrine. The chromosome-breaking potential of orphenadrine was not examined, yet Nielsen et al. attributed the positive effect seen to perphenazine, not considering any possible effects of orphenadrine or a synergism between the two agents administered. Orphenadrine was not studied in the present experiment since its administration was not clinically justifiable. The lack of chromosome damage induced by perphenazine, however, suggests that orphenadrine may have been the active agent in the studies of Nielsen et al. (1969). On the other hand the present studies confirmed the previous negative studies of others concerning chlorpromazine. Cohen et al. (1969) studied this drug in vitro and in vivo with negative results, as did Sandberg in a double-blind study of 35 schizophrenic patients (personal communication). Therefore none of the psychotropic drugs tested for the induction of cytogenetic damage has clearly shown such potential.

These studies were supported in part by grants from the Department of Health Education and Welfare, Maternal and Child Health Service (project No. 417), and the U.S. Public Health Service (MH-05101). We wish to thank Dr. A. Sugarman, New Jersey Psychiatric Institute, Princeton, New Jersey, for allowing the study of patients under his care and for supplying blood specimens, Mr. M. Miller, Schering Corporation, for statistical analysis, and Dr. P. Reyes, Mrs. C. Lockwood, and Mrs. V. Wolff for technical help.

\section{References}

Cohen, M. M., Hirschhorn, K., and Frosch, W. A. (1967). New England fournal of Medicine, 277, 1043

Cohen, M. M., Hirschhorn, K., and Frosch, W. A. (1969). Fournal of the American Medical Association, 207, 2425.

Moorhead, P. S., Nowell, P. C., Mellman, W. J., Battips, D. M., and Hungerford, D. A. (1960). Experimental Cell Research, 20, 613.

Nielsen, J., Friedrich, U., and Tsuboi, T. (1969). British Medical fournal 3, 634 .

Schmid, W., and Staiger, G. R. (1969). Mutation Research, 7, 99.

Schmid, W., and Staiger, G. R. (1969). Mutation R.

Stenchever, M. A., and Frankel, R. B. (1969). American fournal of Obstetrics and Gynecology, 103, 836 .

\title{
Clinical Evaluation of Co-trimoxazole and Furazolidone in Treatment of Shigellosis in Children
}

\author{
UDOM LEXOMBOON, PETHAI MANSUWAN, CHIRAPHUN DUANGMANI, PANYASRI BENJADOL \\ M. TALMAGE M'CMINN
}

British Medical fournal, 1972, 3, 23-26

\section{Summary}

Co-trimoxazole (trimethoprim-sulphamethoxazole) was compared with furazolidone in the treatment of shigellosis in two groups of 33 and 30 patients respectively. Those treated with co-trimoxazole recovered more quickly; none had shigellae in the faeces four days after the start of treatment, whereas in the group given furazolidone eight still had positive stool cultures seven days after treatment.

The susceptibility of 104 shigella strains to seven antimicrobial agents was studied by plate dilution

Children's Hospital, Bangkok, Thailand

UDOM LEXOMBOON, M.B., PH.D., Senior Staff

PETHAI MANSUWAN, M.B., Director

Department of Bacteriology and Mycology, S.E.A.T.O. Medical Research Laboratory, Bangkok, Thailand

CHIRAPHUN DUANGMANI, M.B., Medical Research Specialist

PANYASRI BENIADOL, M.S., Medical Research Technologist

M. TALMAGE M'CMINN, C.P.T., M.S.C., Chief of Department technique. All agents but tetracycline and chloramphenicol were found highly effective against most of the strains tested. All shigella isolates were resistant to sulphamethoxazole, and $63 \%$ were sensitive to trimethoprim. Potentiation of trimethoprim by sulphamethoxazole was shown in that all strains tested became sensitive to the combination of trimethoprim and sulphamethoxazole in a ratio of $1: 20$.

\section{Introduction}

Shigella spp. are the most common bacterial pathogens isolated from diarrhoeal children in many countries (Ramos-Alvarez and Olarte, 1964; Goodwin et al., 1967; Lexomboon and Sunakorn, 1968-9). In a recent study conducted by us at the Children's Hospital, Bangkok, shigella bacilli were found to be the most frequent cause of gastroenteritis in 588 children, with the isolation rate of $20 \%$ (Lexomboon and Sunakorn, 1968-9). Patients with clinical gastroenteritis due to shigellae should be given specific antimicrobial therapy, since several studies have indicated that effective antibiotic treatment rapidly rids the 